\title{
Endothelial microvascular networks affect gene-expression profiles and osteogenic potential of tissue-engineered constructs
}

Torbjorn O Pedersen ${ }^{1,2^{*}}$, Anna L Blois ${ }^{2,3}$, Zhe Xing ${ }^{1}$, Ying Xue ${ }^{1}$, Yang Sun ${ }^{4}$, Anna Finne-Wistrand ${ }^{4}$, Lars A Akslen ${ }^{3,5}$, James B Lorens ${ }^{2}$, Knut N Leknes $^{1}$, Inge Fristad ${ }^{1}$ and Kamal Mustafa ${ }^{1}$

See related commentary by Brennan et al., http://stemcellres.com/content/4/4/96

\begin{abstract}
Introduction: A major determinant of the potential size of cell/scaffold constructs in tissue engineering is vascularization. The aims of this study were twofold: first to determine the in vitro angiogenic and osteogenic geneexpression profiles of endothelial cells (ECs) and mesenchymal stem cells (MSCs) cocultured in a dynamic 3D environment; and second, to assess differentiation and the potential for osteogenesis after in vivo implantation.

Methods: MSCs and ECs were grown in dynamic culture in poly(L-lactide-co-1,5-dioxepan-2-one) (poly(LLA-co-DXO)) copolymer scaffolds for 1 week, to generate three-dimensional endothelial microvascular networks. The constructs were then implanted in vivo, in a murine model for ectopic bone formation. Expression of selected genes for angiogenesis and osteogenesis was studied after a 1-week culture in vitro. Human cell proliferation was assessed as expression of ki67, whereas a-smooth muscle actin was used to determine the perivascular differentiation of MSCs. Osteogenesis was evaluated in vivo through detection of selected markers, by using real-time RT-PCR, alkaline phosphatase (ALP), Alizarin Red, hematoxylin/eosin (HE), and Masson trichrome staining.

Results: The results show that endothelial microvascular networks could be generated in a poly(LLA-co-DXO) scaffold in vitro and sustained after in vivo implantation. The addition of ECS to MSCs influenced both angiogenic and osteogenic gene-expression profiles. Furthermore, human ki67 was upregulated before and after implantation. MSCs could support functional blood vessels as perivascular cells independent of implanted ECs. In addition, the expression of ALP was upregulated in the presence of endothelial microvascular networks.

Conclusions: This study demonstrates that copolymer poly(LLA-Co-DXO) scaffolds can be prevascularized with ECs and MSCs. Although a local osteoinductive environment is required to achieve ectopic bone formation, seeding of MSCs with or without ECs increases the osteogenic potential of tissue-engineered constructs.
\end{abstract}

Keywords: Tissue engineering, Endothelial cells, Mesenchymal stem cells, Copolymer, Osteogenesis

\section{Introduction}

In tissue engineering, the reconstruction of bone defects by using stem cells seeded onto biodegradable carrier materials requires timely formation of functional blood vessels. After in vivo implantation, complex tissues are dependent on a functional vasculature, not only for cell survival, but also for tissue organization. In several

\footnotetext{
* Correspondence: torbjorn.pedersen@iko.uib.no

'Department of Clinical Dentistry, Center for Clinical Dental Research, University of Bergen, Årstadveien 19, Bergen N-5009, Norway

2 Department of Biomedicine, University of Bergen, Bergen, Norway

Full list of author information is available at the end of the article
}

recent review articles, vascularization was highlighted as a major determinant of the potential size of cell/scaffold constructs [1-3]. The osteogenic potential of bone marrow was demonstrated by Friedenstein et al. [4] many years ago. It is suggested that mesenchymal stem cells (MSCs) derived from bone marrow differentiate preferentially along the osteogenic lineage [5]. It is well documented that MSCs can differentiate into skeletal cell types, such as osteoblasts (OBs), chondrocytes, adipocytes, and fibroblasts. Various reports have also found that MSCs possess greater differentiation potential,

\section{Ciomed Central}


including differentiation into nonmesodermal cell types. For review, see [6].

The establishment of a biological vasculature within a tissue-engineered construct influences differentiation of cells present and, subsequently, development of the tissue [7]. In the process of bone formation, vascular endothelial cells (ECs) are intimately associated with osteogenic cells, and ECs are regarded as important regulators of osteogenic differentiation [8,9]. The addition of ECs to osteogenic cells has been shown to cause increased bone formation in calvarial defects as well as after subcutaneous implantation $[8,10]$. Prevascularization, in which three-dimensional EC microvascular networks are developed in vitro through coculture of ECs and MSCs before in vivo implantation, is therefore an approach whereby the phenotype of MSCs delivered to tissue defects might be altered.

The osteoinductive properties of scaffold materials are also important in optimizing constructs for cell-based tissue regeneration. Several authors have shown that various scaffolding materials, including polymer, can induce ectopic bone formation $[8,11,12]$. In vitro findings have also suggested that poly(LLA-co-DXO) scaffolds enhance differentiation of osteogenic cells [13].

In the present study, ECs and MSCs were cocultured onto three-dimensional copolymer scaffolds. The aim was to investigate how the creation of microvascular networks affect the angiogenic and osteogenic geneexpression profiles. A murine model for ectopic bone formation was used to assess further the cellular differentiation and the potential for osteogenesis after in vivo implantation.

\section{Methods}

\section{Cell culture}

Human umbilical vein endothelial cells (ECs) were purchased from Lonza (Clonetics, Walkersville, MD, USA). According to the manufacturer's instructions, ECs were expanded in Endothelial Cell Growth Medium (EGM) (Lonza) containing $500 \mathrm{ml}$ Endothelial Cell Basal Medium and supplements: FBS, $10 \mathrm{ml}$; BBE, $2 \mathrm{ml}$; hEGF, $0.5 \mathrm{ml}$; hydrocortisone, $0.5 \mathrm{ml}$; and GA-1000, $0.5 \mathrm{ml}$. From StemCell Technologies (Vancouver, BC, Canada) primary human bone marrow-derived stem cells (MSCs) were purchased, and expanded in MesenCult complete medium (StemCell Technologies). Flow cytometry was performed to investigate the purity of the cells; it was found that $>90 \%$ of the cells expressed CD29, CD44, CD105, and CD166, whereas $<1 \%$ expressed CD14, CD34, and CD45. Cells no older than passage five were used, and all cells were cultured at $37^{\circ} \mathrm{C}$ and $5 \% \mathrm{CO}_{2}$. To simplify imaging, retroviral transfection of ECs at an early passage with green fluorescent protein (GFP) was performed [14]. Pulmonary artery smooth muscle cells
(SMCs) were purchased from Lonza and expanded in Smooth Muscle Growth Medium-2 (SmGM-2) (Lonza), according to the manufacturer's instructions. MSCs and SMCs grown in vitro were stained with mouse antihuman $\alpha$-smooth muscle actin ( $\alpha$-SMA) (Santa Cruz Biotechnology, Santa Cruz, CA, USA) incubated in a 1:200 dilution for 4 hours at room temperature with Alexa594-conjugated goat anti-mouse IgG as secondary antibody diluted 1:3,000 for 2 hours.

\section{Preparation of cell-seeded scaffolds}

Fabrication of poly(LLA-co-DXO) scaffolds was described thoroughly in previous publications [15,16], and scaffolds seeded with cells for in vivo implantation were prepared in a similar way to the one described by Xing et al. [10]. In brief, the scaffolds were prewet with MesenCult complete medium (StemCell Technologies) and incubated overnight at $37^{\circ} \mathrm{C}$ and in $5 \% \mathrm{CO}_{2}$. Then, $5 \times 10^{5}$ cells were seeded per scaffold, either MSCs alone or MSCs/ECs in a 5:1 ratio. To facilitate distribution of cells, an orbital shaker (Eppendorf, Germany) was used, and cells were allowed to attach overnight before scaffolds were transferred to separate modified spinner flasks (Wheaton Science, Millville, NJ, USA) for 1 week in a dynamic culture system with 50 rotations per minute. MesenCult complete medium without angiogenic or osteogenic supplements was used for both experimental groups. At 1 week, 6-mm discs were punctured from the scaffolds by using a dermal skin puncher and either processed for in vitro analysis or implanted in vivo.

\section{Surgical procedures}

All animal experiments were approved by the Norwegian Animal Research Authority and conducted according to the European Convention for the Protection of Vertebrates used for Scientific Purposes, with local approval numbers 3029 and 1572. For subcutaneous implantation, 16 nonobese severe combined immunodeficient (NOD/ SCID) mice (Gade Institute/Taconic Farms) were used. Animals were aged 6 to 8 weeks at the time of the operation. An intramuscular injection of $20 \mu \mathrm{l}$ of Rompun (xylazine) $(20 \mathrm{mg} / \mathrm{ml})$ (Bayer Health Care, Leverkusen, Germany) and Narketan (ketamine) (Vétoquinol, Lure, France) in a 1:2 ratio was given to anesthetize the animals. On the backs of the mice, a $2.5-\mathrm{cm}$ incision was made, providing sufficient space for subcutaneous implantation of scaffolds. Wounds were closed with Vetbond Tissue Adhesive ( $n$-butyl cyanoacrylate) (3M, St. Paul, MN, USA). Animals were euthanized with deep isoflurane (Schering Plough, Kenilworth, NJ, USA) anesthesia and subsequent cervical dislocation at 1 and 3 weeks. Scaffolds were given careful biopsies and fixated. 
As a positive control, 12 3-month-old Lewis rats were operated on in a model described previously [10]. In brief, cell/scaffold constructs were implanted into $6-\mathrm{mm}$ calvarial bone defects, and the animals were euthanized and samples retrieved after 8 weeks.

\section{Real-time RT-PCR}

RNA was extracted by using an E.Z.N.A. Total RNA Kit (Omega Bio-Tek, Norcross, GA, USA). Quantification and determination of RNA purity was performed with a Nanodrop Spectrophotometer (ThermoScientific NanoDrop Technologies, Wilmington, DE, USA). A highcapacity cDNA Archive Kit (Applied Biosystems, Carlsbad, CA, USA) was used for the reverse-transcription reaction. Total RNA (1,000 ng) was mixed with nuclease-free water, reverse transcriptase buffer, random primers, dNTP, and MultiScribe reverse transcriptase. Real-time RT-PCR was performed on a StepOne real-time PCR system (Applied Biosystems). Standard enzyme and cycling conditions were used, with cDNA corresponding to $10 \mathrm{ng}$ mRNA in each reaction, prepared in duplicates for each target gene. Taqman gene-expression assays were human ki67, human and mouse CD31, human and mouse vascular endothelial growth factor (VEGF), human and mouse $\alpha$-SMA, mouse alkaline phosphatase (ALP), mouse osteopontin $(\mathrm{OP})$, and mouse collagen I (COL I). Data analysis was performed with a comparative $\mathrm{Ct}$ method with $\mathrm{GAPDH}$ as endogenous control.

For superarray analysis of angiogenesis and osteogenesis, $\mathrm{Rt}^{2}$ Profiler PCR Arrays (SuperArray Bioscience, Frederick, MD, USA) were used. $\mathrm{Rt}^{2}$ PCR array First Strand Kit (SuperArray Bioscience) was used for cDNA synthesis, and PCR was performed on a StepOne realtime PCR system (Applied Biosystems), with $\mathrm{Rt}^{2}$ Realtime SyBR Green/Rox PCR mix (SuperArray Bioscience).

\section{Histologic evaluation}

Samples intended for cryosectioning were immediately frozen in O.C.T. tissue-tech (Sakura Finetek, Tokyo, Japan), by using 2-methylbutan ReagentPlus (Sigma-Aldrich, St. Louis, MO, USA) and liquid nitrogen. Cryosectioning was performed on a Leica CM 3050S (Leica Microsystems, Wetzlar, Germany) at $-24^{\circ} \mathrm{C}$ into $8-\mu \mathrm{m}$ sections. Samples intended for paraffin sectioning were fixated in $4 \%$ PFA before embedding. Sections acquired from the middle parts of the samples were stained with CD31 mouse anti-human primary antibody (BD Biosciences, San Jose, CA, USA) or vimentin mouse anti-human primary antibody (Santa Cruz Biotechnology, Santa Cruz, CA, USA) diluted 1:200 in PBS with $5 \%$ goat serum. FITC-conjugated goat anti-mouse (Invitrogen) was used as secondary antibody (1:1,000). DAPI was used for nuclear staining $(1: 3,000)$ for 2 minutes at room temperature. Double staining with TRITCconjugated Ulex europaeus Agglutinin (UEA-1) and CD31 was performed to evaluate the interaction between implanted human vessels and the host circulation. All vessels were stained with UEA-1 (Sigma-Aldrich) (diluted 1:500) by incubation for 2 hours at room temperature protected from light. Human vessels were stained as previously described. Scaffolds were autofluorescent with DAPI filter.

Staining for $\alpha$-SMA was done on $5-\mu \mathrm{m}$ sections of formalin-fixed and paraffin-embedded scaffolds. The sections were deparaffinized with xylene and rehydrated in decreasing concentrations of ethanol to water. Pretreatment of the sections was done by using antigen retrieval at $57^{\circ} \mathrm{C}$ overnight in Target Retrieval Solution, $\mathrm{pH}$ 6.0 (Dako, Glostrup, Denmark) after 8-minute incubation with Dual Endogenous Enzyme-Blocking Reagent (Dako). Sections were incubated for 30 minutes at room temperature with monoclonal mouse anti-human $\alpha$-SMA (M0851; Dako) diluted 1:200. Rat-anti-Mouse-IgG2a/AP secondary antibody was used, and $\alpha$-SMA visualized by incubating with the Ferangi Blue Chromogen Kit (Biocare Medical, Concord, CA, USA) for 20 minutes at room temperature. Antibody specificity was validated by using negative-control sections containing mouse vascular tissue and positive-control sections containing human vascular tissue.

Alkaline phosphatase staining was performed with freshly made substrate solution (Sigma-Aldrich) containing $100 \mathrm{~m} M$ Tris-maleate buffer, $8 \mathrm{mg} / \mathrm{ml}$ Naphthol AS-TR, and $2 \mathrm{mg} / \mathrm{ml}$ Diazoniumsalt Fast Red Violet LB. Slides were incubated for 2 hours at room temperature, before washing with distilled water and counterstaining with $0.1 \%$ fast green. Alizarin Red staining was performed with $2 \%$ Alizarin Red powder (Sigma-Aldrich) dissolved in distilled water, with $\mathrm{pH}$ adjusted to 4.2 with $0.5 \%$ ammonium hydroxide for 20 minutes at room temperature. A graded alcohol and xylol series was applied before mounting with eukitt (O. Kindler, Freiburg, Germany). Alizarin Red staining was quantified from three sections at $10 \times$ magnification for each sample, covering the area of the sections. The total sample size was four mice for each group. A common threshold was applied to all images, identifying highdensity areas of red staining by using NIS-Elements BR 3.07 (Nikon, Tokyo, Japan) with a resulting quantified total area fraction of calcified nodules. Masson trichrome and HE staining was performed on in vivo samples.

A Nikon 80i microscope (Nikon) was used for light microscopy, and confocal images were acquired on a Zeiss LSM 510 Meta (Carl Zeiss, Oberkocken, Germany). All sections stained with fluorescent secondary antibodies were mounted with Prolong Gold Antifade Reagent (Invitrogen) before imaging.

\section{Statistical analysis}

PCR data presented are from six parallel samples. Groups with monocultured MSCs or MSCs/ECs were 
compared with the independent samples $t$ test, in which $P<0.05$ was defined as a significant difference. SPSS Statistics 19.0 (IBM, Armonk, NY, USA) was applied for statistical processing and analysis.

\section{Results}

In EC/MSC constructs, endothelial microvascular networks were observed after 1 week of dynamic culture in vitro (Figure $1 \mathrm{~A}$, left). Moreover, 1 week after in vivo implantation, ECs positive for human CD31 were organized into networks (Figure 1A, middle) and tubular structures (Figure 1A, right). In monocultured MSCs, no human endothelial microvascular structures were detected (not shown). Incorporation of implanted human ECs with the surrounding vascular bed was observed through double staining for human and mouse blood vessels (Figure 1B). As expected, gene-expression levels of human CD31 were higher in cocultured constructs at 1 and 3 weeks of implantation and did not subside during the experimental period (Figure 1C).

Gene-expression profiles were evaluated after 1 week of dynamic culture, before the cell/scaffold constructs were implanted in vivo. Evaluation of genes related to angiogenesis showed that in all, 37 genes were downregulated, and six genes were upregulated, with a fold change more than three in the MSC/EC group, compared with monocultured MSCs (Figure 2A). Functional grouping showed that genes related to skeletal development and the extracellular matrix (ECM), were expressed predominantly in monocultured MSCs, whereas higher expression of genes was found related to cell growth and differentiation in cocultures (Table 1). The mRNA expression of VEGF of mouse origin was higher in monocultured constructs at 1 week, although not statistically significantly. At 3 weeks, the expression was similar for both experimental groups (Figure 2B). VEGF from the implanted human cells showed the opposite tendency, with upregulation in cocultured constructs at 3 weeks (Figure 2C). However, when we analyzed the expression of mouse CD31, only minor differences were found during the experimental period, suggesting a similar effect from both constructs on the ingrowth of host ECs (Figure 2D).

Perivascular $\alpha$-SMA was used as a biomarker to assess maturation of developing mouse vasculature into the

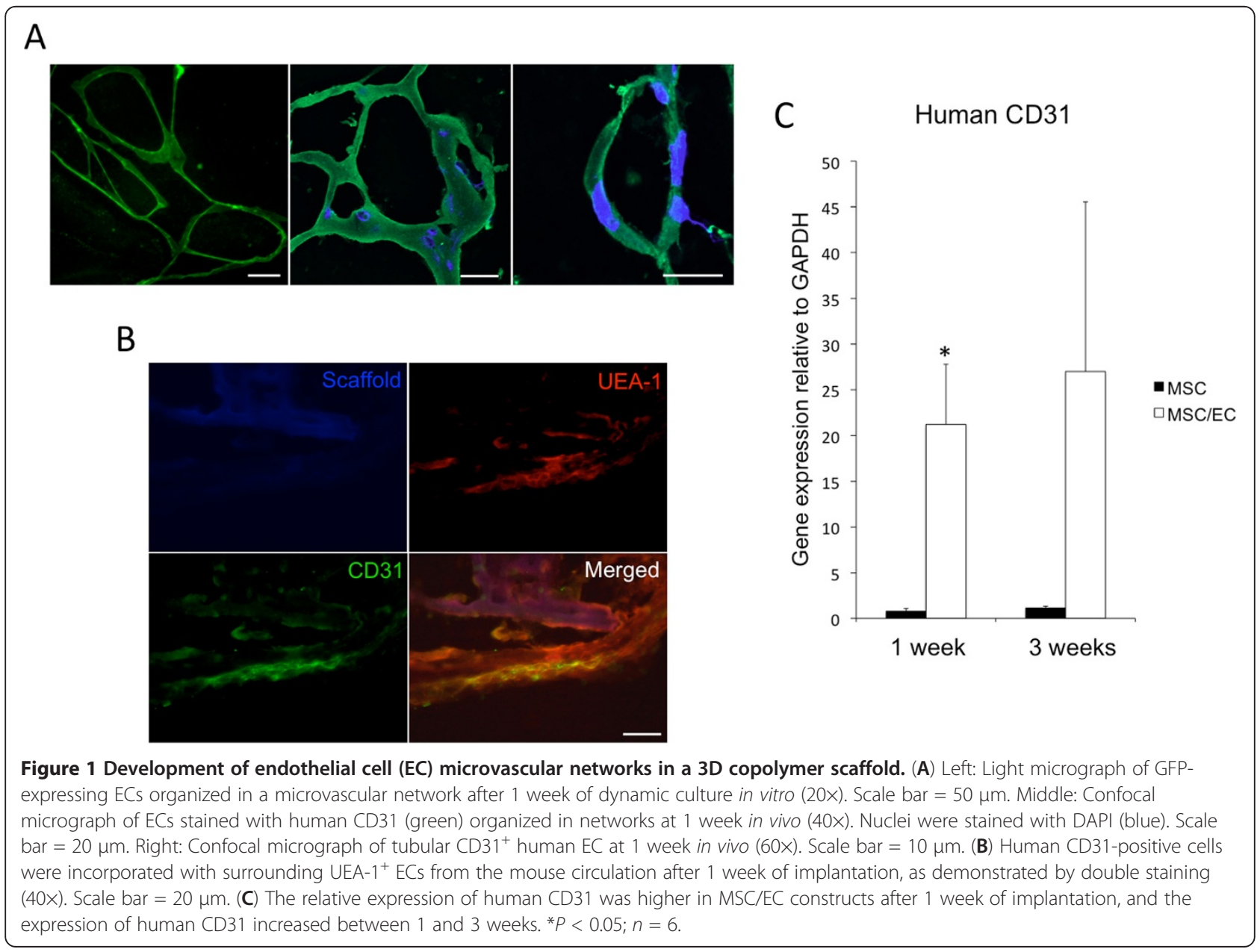




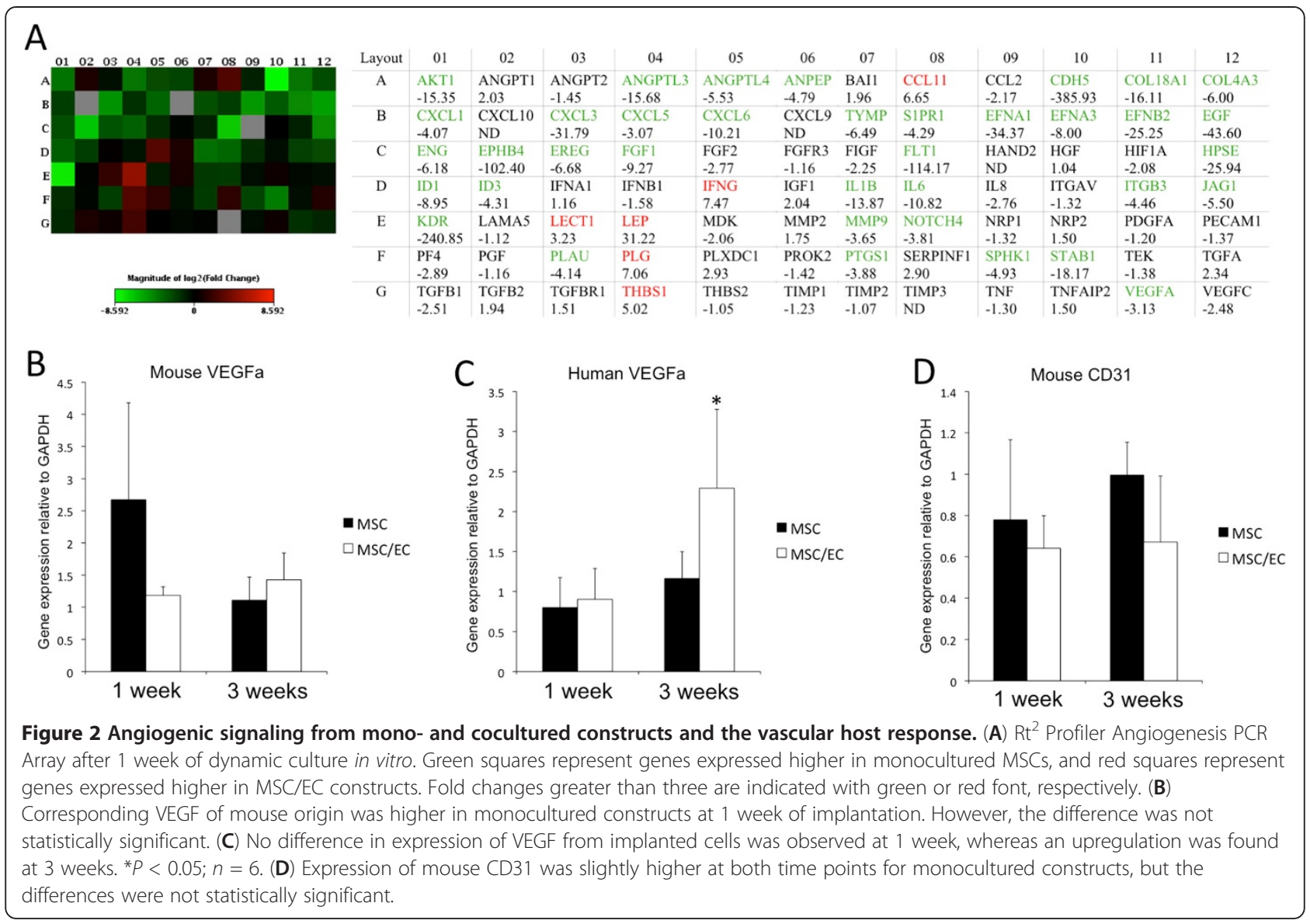

scaffolds. An upregulation of mouse $\alpha$-SMA was observed at 3 weeks compared with 1 week, but no difference was detected between the groups (Figure 3A, left). The expression of human $\alpha$-SMA was higher in the MSC/EC group at 1 week of culture in vitro, and at 1 and 3 weeks in vivo (Figure 3A, right). However, the differences were not statistically significant. Interestingly, when compared with control scaffolds in which no cells were implanted, a strong downregulation of mouse $\alpha$ SMA was observed for both mono- and cocultured constructs (Figure 3B). Positive staining for human $\alpha-S M A$ was found in both groups, surrounding functional blood vessels identified by the presence of red blood cells in the lumen (Figure $3 \mathrm{C}$ ). When evaluating MSCs in vitro for expression of $\alpha$-SMA, positive staining comparable to that of SMCs was observed for cells grown in standard culture conditions, showing that undifferentiated MSCs express $\alpha$-SMA (Figure 3D). These findings suggest a perivascular role for MSCs in the development of a functional microvasculature in vivo.

The osteogenic potential was evaluated at 3 weeks after implantation, and positive staining for ALP and Alizarin Red was observed for both experimental groups (Figure 4A). Gene expression of ALP was upregulated in cocultured constructs when compared with both empty controls and monocultured constructs. However, only the former comparison was statistically significant. Osteogenic biomarkers OP and COL I were both similarly expressed in the two groups (Figure 4B). Figure $4 \mathrm{C}$ shows the total area of calcification as positive Alizarin Red staining, which was higher for both cellular constructs when compared with empty controls, where no positive staining was found. Only minor differences were observed between the two experimental groups.

Human ki67 was used as a biomarker for proliferation of human cells, and the relative gene expression was evaluated before in vivo implantation and at 1 and 3 weeks in vivo. Human ki67 was significantly upregulated $(P<0.05)$ in the MSC/EC constructs before implantation and at 1 week. At 3 weeks, a difference was still observed, but was not statistically significant (Figure 5A).

After 3 weeks of subcutaneous implantation, scaffolds from both groups exhibited penetration with normal vascularized loose connective tissue (Figure 5B, upper). The mesenchymal cell marker vimentin was used to evaluate the presence of human cells in vivo, and cells positive for human vimentin were detected in both groups (Figure 5C, upper). Masson trichrome stain was 
Table 1 Functional grouping of osteogenic gene expression after 1 week of dynamic culture in vitro

\begin{tabular}{lll}
\hline Gene & Full name & Fold change \\
\hline \multicolumn{3}{c}{ Skeletal development } \\
\hline AHSG & Alpha-2-HS-glycoprotein & -3.7044 \\
\hline AMBN & Ameloblastin & -4.9367 \\
\hline AMELY & Amelogenin & -4.68 \\
\hline ENAM & Enamelin & -2.8164 \\
\hline STATH & Statherin & -9.6485 \\
\hline ALPL & Alkaline phosphatase & -3.6727 \\
\hline CALCR & Calcitonin receptor & -2.9072 \\
\hline DMP1 & Dentin matrix acidic phosphoprotein 1 & -3.9158 \\
\hline BMP3 & Bone morphogenetic protein 3 & -4.5315 \\
\hline BMP5 & Bone morphogenetic protein 5 & -4.9367 \\
\hline COL2A1 & Collagen type II, alpha 1 & -3.373 \\
\hline & \multicolumn{1}{c}{ Cell growth and differentiation } \\
\hline IGF1R & Insulin-like growth factor 1 receptor & \\
\hline IGF2 & Insulin-like growth factor 2 & 3.5697 \\
\hline TGFB3 & Transforming growth factor beta 3 & 4.9403 \\
\hline IGF1 & Insulin-like growth factor 1 & 2.2567 \\
\hline BMP2 & Bone morphogenetic protein 2 & 2.964 \\
\hline GDF10 & Growth differentiation factor 10 & 2.476 \\
\hline TWIST1 & Twist homolog 1 & 2.2778 \\
\hline FLT1 & Fms-related tyrosine kinase 1 & 2.1232 \\
\hline MMP10 & Matrix metallopeptidase 10 & -8.1751 \\
\hline CSF2 & Colony-stimulating factor 2 & -5.3112 \\
\hline ITGAM & Colony-stimulating factor 3 & -4.9367 \\
\hline Integrin alpha M & -3.9367 \\
\hline Collagen type XIV, alpha 1 & 2.7336 \\
\hline & Cathepsin K & \\
\hline & & \\
\hline
\end{tabular}

negative for osteoid formation (Figure 5B, lower), which was evident in cell/scaffold constructs implanted into calvarial bone defects (Figure 5C, lower).

\section{Discussion}

This study evaluated the potential for ECs in combination with MSCs to create microvascular networks in three-dimensional bone tissue engineered constructs. To assess how coculture of MSCs with ECs influenced the phenotype of cells delivered for tissue regeneration in vivo, we studied angiogenic and osteogenic geneexpression profiles after 1 week of dynamic culture in vitro. The results show that formation of an endothelial microvascular network resulted in upregulated expression of human ki67, a well-described biomarker for cellular proliferation [17]. Various genes involved in cell growth and differentiation, were upregulated in MSC/EC constructs, and the influence of ECs on both angiogenic and osteogenic gene-expression profiles was evident.
The results of the in vivo experiment showed that the response to the angiogenic signal on ingrowth of CD31positive cells was similar for both experimental groups, but that implanted human MSCs could support functional blood vessels as perivascular cells. Histologic evaluation showed that generation of ectopic bone in poly(LLA-coDXO) scaffolds required a local osteoinductive environment. However, increased osteogenic potential was found for both cellular constructs compared with empty controls, and expression of ALP was significantly upregulated in the presence of endothelial microvascular networks.

The potential use of biodegradable polymers as scaffolds for cell-based tissue regeneration was reviewed by Gunatillake and Adhikari [18]. An important attribute of these materials is their chemical versatility, allowing mechanical properties and material degradation to be tailored to specific clinical conditions. Hence these materials are potentially applicable to regeneration of various types of tissue. The response of both OBs and MSCs to the scaffold used in this report has been investigated in several in vitro studies $[13,15,19]$. The results from these studies suggest that the material enhances osteogenic differentiation of both cell types. Our results show that the osteogenic stimulatory effects of the scaffold material and the cellular interactions are not sufficient to induce ectopic bone formation in NOD/SCID-mice. However, several other authors have used polymer scaffolds to deliver osteogenic growth factors and then induce ectopic bone formation [20,21]. MSC/polymer constructs, cultured with or without osteogenic stimulatory conditions before implantation, have also been investigated for their osteogenic potential, with successful generation of ectopic bone [8,22].

Complex tissues depend on functional blood vessels for cell survival as well as for tissue organization after in vivo implantation. Prevascularization, in which endothelial cell microvascular networks are developed in vitro, have been attempted with different cells and materials. Microvascular networks were created by using MSC/EC coculture spheroids, in which limited functionality was found in vivo [23]. Asakawa et al. [24] were able to generate a three-dimensional tissue by using dermal fibroblasts as supporting cells for developing endothelial microvascular networks, whereas $\mathrm{Yu}$ et al. [25] seeded ECs with OBs in poly- $\varepsilon$-caprolactone and hydroxyapatite scaffolds, and subsequently demonstrated enhanced osteogenesis in rat long-bone defects. Thus, for development of a de novo microvasculature, ECs depend on supporting cells, a role that can be undertaken by several cell types $[26,27]$. Our results show that a poly(LLA-co-DXO) scaffold can support the formation of EC/MSC microvascular networks in three dimensions, suggesting that prevascularized tissue regeneration with this material is feasible. 


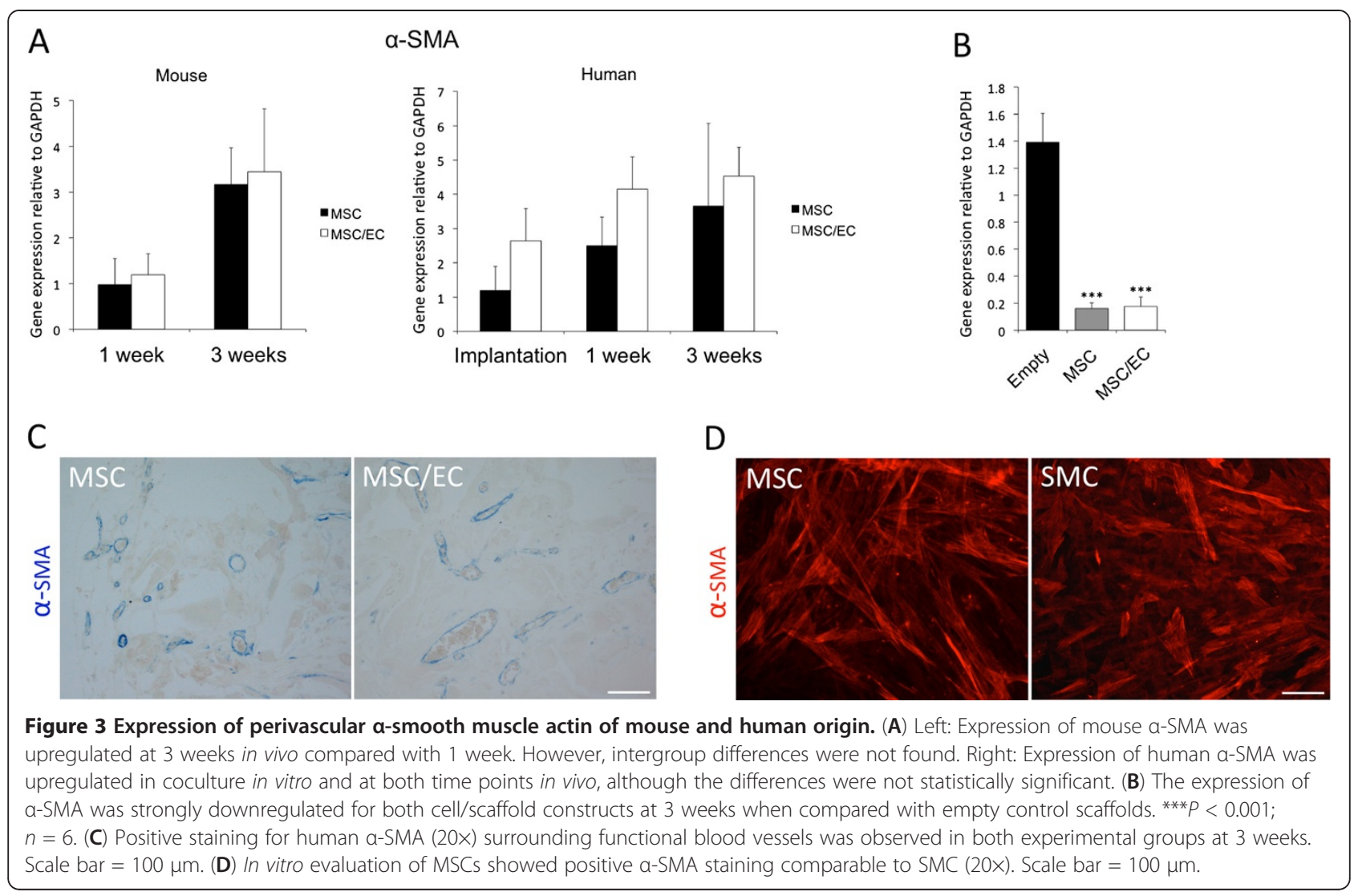

In the present study, we cocultured cells for 1 week before in vivo implantation and found altered geneexpression profiles with reference to both angiogenesis and osteogenesis. Genes related to skeletal development and the ECM were highly expressed in monocultured MSCs. These results are in agreement with an earlier study by Fuchs et al. [28], showing that the expression of osteogenic markers from OBs was downregulated in the presence of ECs in 4-week cultures. Long-term incubation in vitro is beneficial when studying the mineralization process, but might be considered less practical for clinical applications and have less potential for regenerating bone [29]. Although an overview of osteogenic and angiogenic gene-expression profiles were generated in the present work, functional evaluations of candidate targets might have been of interest to assess further the effect from regulation of individual genes. Evaluations on tissue development were made on the gene, protein, and morphologic level, to determine the effect of the paracrine signal from all biologic factors delivered by the tissue-engineered constructs.

Murine models of ectopic bone formation are widely used to evaluate osteogenic potential in bone-tissue engineering. By using a subcutaneous mouse model to compare implantation of MSCs and OBs, Tortellini et al. [30] reported that MSCs enhanced vascularization through increased recruitment of host ECs. MSCs are known to secrete multiple paracrine factors stimulating EC migration and wound healing [31], and this might be downregulated when ECs are already present in the construct. In the present study, angiogenic gene expression was higher in monocultured MSCs at the moment of implantation, suggesting that increased angiogenic stimulation could be delivered to the host bed from MSCs alone. With ECs already present, paracrine signals to attract and activate ECs might be less relevant in the coculture system.

An initial response from the host circulation was also found as higher expression of mouse VEGF at 1 week, when compared with cocultured constructs. The opposite regulation of human VEGF was observed, with upregulation in the coculture group at the end of the experimental period. These findings might be interpreted as cell/scaffold constructs containing ECs having a stronger ability to maintain a proangiogenic signal after implantation. However, the expression of mouse CD31 was not notably different for the two experimental groups, suggesting a similar vascular host response with regard to the presence of ECs. The total number of blood vessels was not quantified, but no obvious difference could be observed.

Recruitment of perivascular cells with subsequent production of basement membrane proteins are key events 

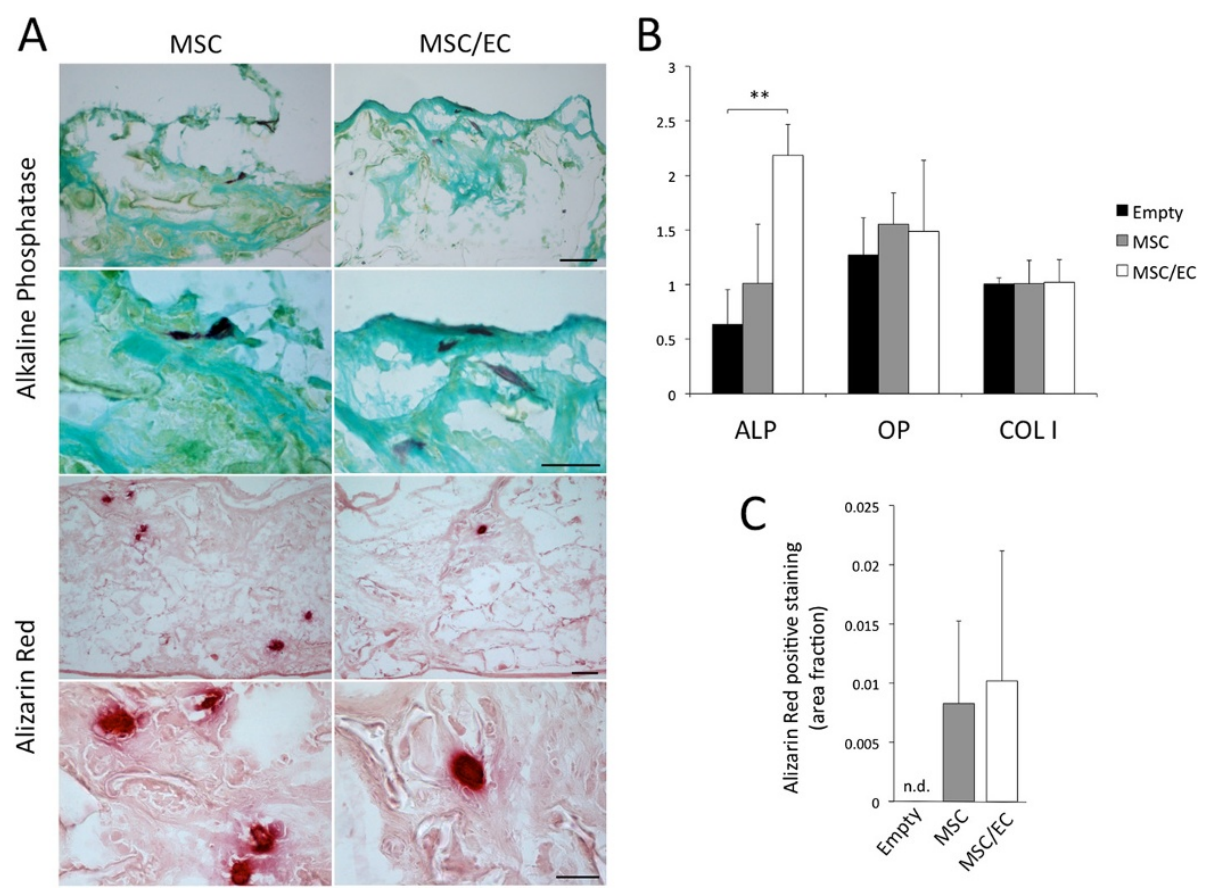

Figure 4 Osteogenic potential of tissue-engineered constructs after 3 weeks of subcutaneous implantation. (A) Positive staining for alkaline phosphatase (purple) could be observed for both cell/scaffold constructs, as shown at 10x and 60x magnification. Scale bar: upper $=100$ $\mu \mathrm{m}$, lower $=20 \mu \mathrm{m}$. Calcified nodules were observed as positive Alizarin Red staining (red) shown at $4 \times$ and 40x magnification. Scale bar: upper $=100 \mu \mathrm{m}$, lower $=20 \mu \mathrm{m}$. (B) Gene-expression levels of osteogenic biomarkers alkaline phosphatase (ALP), osteopontin (OP), and collagen I (COL I) were evaluated with real-time RT-PCR, and increased expression of ALP was detected for MSC/EC constructs compared with empty controls. ${ }^{*} P<0.01 ; n=6$. (C) Quantification of Alizarin Red staining did not reveal significant differences between MSC and MSC/EC constructs. No positive staining was observed in the control scaffolds without cells; $n=4$.

in the maturation of developing vasculature [32]. The $\alpha$ SMA originated from mouse cells was similarly expressed on the mRNA level in MSC and MSC/EC constructs, with an expected increase for both groups between 1 and 3 weeks, as scaffolds were increasingly penetrated with tissue. MSCs in cell/scaffold constructs responded to the presence of ECs by upregulating the expression of $\alpha$-SMA at all time points, although the differences were not statistically significant. In vitro evaluation showed that $\alpha$-SMA was expressed also in

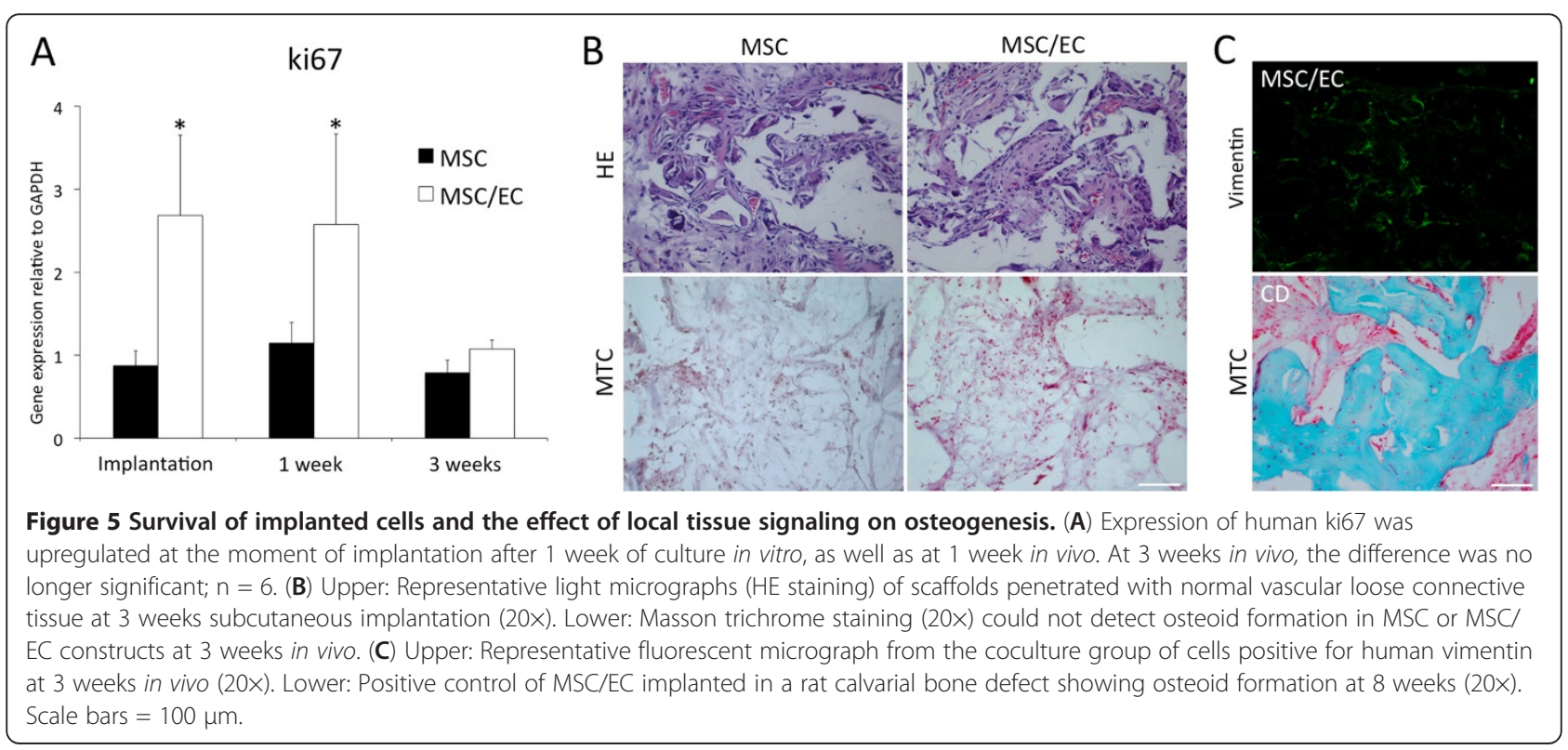


undifferentiated MSCs, whereas the results from the in vivo experiment showed that adding ECs resulted in further upregulation of $\alpha$-SMA. However, the ability of MSCs to function as perivascular cells for developing vessels did not depend on the coimplantation of ECs. Gene expression of $\alpha$-SMA was strongly downregulated for both constructs when compared with empty controls, suggesting less demand for transcription of $\alpha$-SMA after implantation of MSCs. Furthermore, $\alpha$-SMA-positive cells surrounding functional blood vessels were found well distributed in the connective tissue for both experimental groups. In addition to $\alpha$-SMA, positive staining of human vimentin was demonstrated, showing that implanted MSCs were a viable part of the connective tissue.

Kaigler et al. [8] studied dermal microvascular endothelial cells and MSCs seeded on poly(lactic co-glycolic acid) scaffolds implanted subcutaneously in immunodeficient mice. No significant difference was observed in the number of total blood vessels at 2 and 4 weeks, but in line with our findings, a higher percentage of humanderived vessels were found in MSC/EC constructs. At 4 weeks, the area of bone as a percentage of the total tissue area was as high as 35\% in the MSC/EC group. In the present study, we cultured cells for 1 week before implantation in vivo for 3 weeks, but no osteoid formation was detectable within the constructs. We used MSCs at a lower passage, and this might have influenced the differentiation stage of cells at implantation and, subsequently, the osteogenic potential. In addition, the difference in density of cells with regenerative potential should be considered in the interpretation of the results. When evaluating the expression of osteogenic biomarkers, we found an upregulation of ALP for MSC/ECconstructs compared with both monocultured MSCs and empty control scaffolds. These results are in accordance with those of Xue et al. [9] with a two-dimensional coculture MSC/EC model. Compared with monocultured MSCs, at 5 days, multiple osteogenic genes from the cocultured MSC/EC exhibited downregulation, with the exception of ALP [9]. The proposed mechanism for endothelial influence on osteogenic differentiation was therefore that ECs promoted maintenance of MSCs at a proliferative stage rather than inducing terminal differentiation. Although full penetration of tissue within scaffolds was confirmed at the end of the experiment in the present work, longer observation periods might have been of interest to follow further the remodeling and maturation of the tissue.

Positive Alizarin Red staining was found in both cell/ scaffold constructs, but not in scaffolds implanted without cells. The majority of the area within the material was filled with connective tissue, with interspersed calcified nodules. The total area of positive staining was not significantly different for the two experimental groups. Both MSCs and MSC/EC thus enhanced the osteogenic potential of poly(LLA-co-DXO) scaffolds after ectopic implantation.

\section{Conclusions}

The results demonstrate that by using a construct comprising MSCs and ECs seeded onto poly(LLA-co-DXO) scaffolds, we can create three-dimensional microvascular networks in vitro and sustain them in vivo. Furthermore, human MSCs can serve as perivascular cells in the development of functional blood vessels, independent of implanted ECs. The presence of endothelial microvascular networks leads to altered angiogenic and osteogenic gene expression on implantation, and seeding of MSCs with or without ECs increases the osteogenic potential of tissue-engineered constructs.

\section{Abbreviations}

a-SMA: a-smooth muscle actin; ALP: Alkaline phosphatase; COL I: Collagen l; EC: Endothelial cell; ECM: Extracellular matrix; GFP: Green fluorescent protein; MSC: Mesenchymal stem cell; NOD/SCID: Non-obese severe combined immunodeficient mouse; OB: Osteoblast; OP: Osteopontin; poly(LLA-coDXO): Poly(L-lactide-co-1,5-dioxepan-2-one); SMC: Smooth muscle cell; VEGF: Vascular endothelial growth factor.

\section{Competing interests}

The authors declare that they have no competing interests.

\section{Authors' contributions}

TOP, ALB, ZX, YX, KNL, IF, and KM conceived and designed experiments. TOP, ALB, ZX, YX and YS performed experiments. TOP, ALB, ZX, and KM analyzed data. TOP, ALB, KNL, IF, and KM wrote the paper. AFW, LAA, JBL and $\mathrm{KM}$ contributed reagents, materials and analytical tools. All authors read and approved the final manuscript.

\section{Acknowledgements}

The confocal imaging was performed at the Molecular Imaging Center (Fuge, Norwegian Research Council), University of Bergen. This study was supported by the Research Council of Norway; Stem Cell, grant number 180383/N40, and the VascuBone project, European Union FP7; No. 242175. The authors thank Dr Joan Bevenius-Carrick for constructive comments on the manuscript.

\section{Author details}

${ }^{1}$ Department of Clinical Dentistry, Center for Clinical Dental Research, University of Bergen, Årstadveien 19, Bergen N-5009, Norway. ²Department of Biomedicine, University of Bergen, Bergen, Norway. ${ }^{3}$ Centre for Cancer Biomarkers, The Gade Institute, University of Bergen, Bergen, Norway. ${ }^{4}$ Department of Fibre and Polymer Technology, KTH Royal Institute of Technology, Stockholm, Sweden. ${ }^{5}$ Department of Pathology, Haukeland University Hospital, Bergen, Norway.

Received: 5 September 2012 Revised: 13 February 2013 Accepted: 14 May 2013 Published: 17 May 2013

\section{References}

1. Novosel EC, Kleinhans C, Kluger PJ: Vascularization is the key challenge in tissue engineering. Adv Drug Deliv Rev 2011, 63:300-311.

2. Santos Ml, Reis RL: Vascularization in bone tissue engineering: physiology, current strategies, major hurdles and future challenges. Macromol Biosci 2010, 10:12-27.

3. Cancedda R, Giannoni P, Mastrogiacomo M: A tissue engineering approach to bone repair in large animal models and in clinical practice. Biomaterials 2007, 28:4240-4250. 
4. Friedenstein AJ, Piatetzky S II, Petrakova KV: Osteogenesis in transplants of bone marrow cells. J Embryol Exp Morphol 1966, 16:381-390.

5. Bianco P, Robey PG, Simmons PJ: Mesenchymal stem cells: revisiting history, concepts, and assays. Cell Stem Cell 2008, 2:313-319.

6. Charbord P: Bone marrow mesenchymal stem cells: historical overview and concepts. Hum Gene Ther 2010, 21:1045-1056.

7. Rivron NC, Liu JJ, Rouwkema J, de Boer J, van Blitterswijk CA: Engineering vascularised tissues in vitro. Eur Cell Mater 2008, 15:27-40.

8. Kaigler D, Krebsbach PH, West ER, Horger K, Huang YC, Mooney DJ: Endothelial cell modulation of bone marrow stromal cell osteogenic potential. FASEB J 2005, 19:665-667.

9. Xue Y, Xing Z, Hellem S, Arvidson K, Mustafa K: Endothelial cells influence the osteogenic potential of bone marrow stromal cells. Biomed Eng Online 2009, 8:34.

10. Xing Z, Xue Y, Dånmark S, Schander K, Ostvold S, Arvidson K, Hellem S, Finne-Wistrand A, Albertsson AC, Mustafa K: Effect of endothelial cells on bone regeneration using poly(L-lactide-co-1,5-dioxepan-2-one) scaffolds. J Biomed Mater Res A 2011, 96:349-357.

11. Kasten $P$, Vogel J, Luginbühl R, Niemeyer $P$, Tonak $M$, Lorenz $H$, Helbig L, Weiss S, Fellenberg J, Leo A, Simank HG, Richter W: Ectopic bone formation associated with mesenchymal stem cells in a resorbable calcium deficient hydroxyapatite carrier. Biomaterials 2005, 26:5879-5889.

12. Trojani C, Boukhechba F, Scimeca JC, Vandenbos F, Michiels JF, Daculsi G, Boileau P, Weiss P, Carle GF, Rochet N: Ectopic bone formation using an injectable biphasic calcium phosphate/Si-HPMC hydrogel composite loaded with undifferentiated bone marrow stromal cells. Biomaterials 2006, 27:3256-3264

13. Idris SB, Arvidson K, Plikk P, Ibrahim S, Finne-Wistrand A, Albertsson AC, Bolstad Al, Mustafa K: Polyester copolymer scaffolds enhance expression of bone markers in osteoblast-like cells. J Biomed Mater Res A 2010, 94:631-639.

14. Swift S, Lorens J, Achacoso P, Nolan GP: Rapid production of retroviruses for efficient gene delivery to mammalian cells using 293T cell-based systems. In Current Protocols in Immunology, Volume 10. Edited by Coligan JE. New York: John Wiley and Sons; 1999:1-17.

15. Danmark S, Finne-Wistrand A, Wendel M, Arvidson K, Albertsson AC, Mustafa K: Osteogenic differentiation by rat bone marrow stromal cells on customized biodegradable polymer scaffolds. J Bioact Compat Pol 2010, 25:207-223.

16. Odelius K, Plikk P, Albertsson AC: Elastomeric hydrolyzable porous scaffolds: copolymers of aliphatic polyesters and a polyether-ester. Biomacromolecules 2005, 6:2718-2725.

17. Schlüter C, Duchrow M, Wohlenberg C, Becker MH, Key G, Flad HD, Gerdes $\mathrm{J}$ : The cell proliferation-associated antigen of antibody Ki-67: a very large, ubiquitous nuclear protein with numerous repeated elements, representing a new kind of cell cycle-maintaining proteins. J Cell Biol 1993, 123:513-522.

18. Gunatillake PA, Adhikari R: Biodegradable synthetic polymers for tissue engineering. Eur Cell Mater 2003, 5:1-16. discussion.

19. Idris SB, Bolstad Al, Ibrahim SO, Dånmark S, Finne-Wistrand A, Albertsson AC, Arvidson K, Mustafa K: Global gene expression profile of osteoblast-like cells grown on polyester copolymer scaffolds. Tissue Eng Part A 2011, 17:2817-2831.

20. Saito N, Okada T, Horiuchi H, Ota H, Takahashi J, Murakami N, Nawata M, Kojima S, Nozaki K, Takaoka K: Local bone formation by injection of recombinant human bone morphogenetic protein-2 contained in polymer carriers. Bone 2003, 32:381-386.

21. Whang K, Tsai DC, Nam EK, Aitken M, Sprague SM, Patel PK: Ectopic bone formation via rhBMP-2 delivery from porous bioabsorbable polymer scaffolds. J Biomed Mater Res 1998, 42:491-499.

22. Claase MB, de Bruijn JD, Grijpma DW, Feijen J: Ectopic bone formation in cell-seeded poly(ethylene oxide)/poly(butylene terephthalate) copolymer scaffolds of varying porosity. J Mater Sci Mater Med 2007, 18:1299-1307.

23. Rouwkema J, de Boer J, Van Blitterswijk CA: Endothelial cells assemble into a 3-dimensional prevascular network in a bone tissue engineering construct. Tissue Eng 2006, 12:2685-2693.

24. Asakawa N, Shimizu T, Tsuda Y, Sekiya S, Sasagawa T, Yamato M, Fukai F, Okano T: Pre-vascularization of in vitro three-dimensional tissues created by cell sheet engineering. Biomaterials 2010, 31:3903-3909.
25. Yu H, VandeVord PJ, Mao L, Matthew HW, Wooley PH, Yang SY: Improved tissue-engineered bone regeneration by endothelial cell mediated vascularization. Biomaterials 2009, 30:508-517

26. Evensen L, Micklem DR, Blois A, Berge SV, Aarsaether N, Littlewood-Evans A, Wood J, Lorens JB: Mural cell associated VEGF is required for organotypic vessel formation. PLoS One 2009, 4:e5798.

27. Pedersen TO, Blois AL, Xue Y, Xing Z, Cottler-Fox M, Fristad I, Leknes KN, Lorens JB, Mustafa K: Osteogenic stimulatory conditions enhance growth and maturation of endothelial cell microvascular networks in culture with mesenchymal stem cells. J Tissue Eng 2012, 3:2041731412443236.

28. Fuchs S, Jiang X, Schmidt H, Dohle E, Ghanaati S, Orth C, Hofmann A, Motta A, Migliaresi C, Kirkpatrick CJ: Dynamic processes involved in the prevascularization of silk fibroin constructs for bone regeneration using outgrowth endothelial cells. Biomaterials 2009, 30:1329-1338.

29. Castano-Izquierdo H, Alvarez-Barreto J, van den Dolder J, Jansen JA, Mikos AG, Sikavitsas VI: Pre-culture period of mesenchymal stem cells in osteogenic media influences their in vivo bone forming potential. J Biomed Mater Res A 2007, 82:129-138.

30. Tortelli F, Tasso R, Loiacono F, Cancedda R: The development of tissue-engineered bone of different origin through endochondral and intramembranous ossification following the implantation of mesenchymal stem cells and osteoblasts in a murine model. Biomaterials 2010, 31:242-249.

31. Chen L, Tredget EE, Wu PY, Wu Y: Paracrine factors of mesenchymal stem cells recruit macrophages and endothelial lineage cells and enhance wound healing. PLoS One 2008, 3:e1886.

32. Jain RK: Molecular regulation of vessel maturation. Nat Med 2003, 9:685-693.

doi:10.1186/scrt202

Cite this article as: Pedersen et al:: Endothelial microvascular networks affect gene expression profiles and osteogenic potential of tissue-engineered constructs. Stem Cell Research \& Therapy 2013 4:52.

\section{Submit your next manuscript to BioMed Central and take full advantage of:}

- Convenient online submission

- Thorough peer review

- No space constraints or color figure charges

- Immediate publication on acceptance

- Inclusion in PubMed, CAS, Scopus and Google Scholar

- Research which is freely available for redistribution 